\title{
Examination of the heavy-ion collisions using EPOS model in the frame of BES program at RHIC
}

\author{
Maria Stefaniak ${ }^{1, a}$ and Hanna Zbroszczyk ${ }^{1}$ \\ ${ }^{1}$ Warsaw University of Technology, Poland
}

\begin{abstract}
EPOS generator allows one to simulate various types of collisions (nucleinuclei or even more complex ion-ion) with different initial parameters. It is based on Gribov-Regge theory. So far the EPOS model has been used to describe higher collision energies obtained with RHIC ${ }^{b}$ or LHC $^{c}$ data. However, there is another program under investigation: Beam Energy Scan conducted at Brookhaven National Laboratory. The beams of gold ions are collided at energies such as $\sqrt{s_{N N}}-7.7,11.5,19.6,27,39$, and $62.4 \mathrm{GeV}$ in order to collect and analyze data needed to examine QGP Phase Diagram. The EPOS model could become a useful tool in such studies. Simulated with EPOS data will be verified using elliptic flow analyses and two-particles femtoscopic correlations, which allow one to measure the size of sources determined by newly created particle.
\end{abstract}

\section{Introduction}

One way to study the properties of the nuclear matter are collisions heavy-ions in major experiments. Scientists explain such processes in detailed theories. One of such descriptions is included in the phenomenological model EPOS. It considers the pardon-based Gribov-Regge theory [1]. EPOS gives possibility to study different observables what helps to understand even complex reactions with heavyions. So far the EPOS model has been used to describe higher collision energies obtained with RHIC or LHC. However, there is another interesting program currently under investigation at RHIC: Beam Energy Scan (BES), conducted at Brookhaven National Laboratory. Main goals of this project are to examine the Phase Diagram, to study the characteristics of the first-order phase transition between Hadron Gas and Quark-Gluon Plasma phases of nuclear matter, to search signatures of turn-off the QGP. The search of Critical Point between first-order phase transition and transition of "cross-over" is another interesting topic. RHIC collides beams of Au nuclei at selected energies as: $\sqrt{s_{N N}}-$ $7.7,11.5,19.6,27,39$, and $62.4 \mathrm{GeV}$. The variety of initial conditions provides covering as widest part of Phase Diagram of nuclear matter as possible.

\section{EPOS}

Energy conserving quantum mechanical multiple scattering approach, based on Partons (parton ladders), Off-shell remnants, and Splitting of parton ladders [1].

\footnotetext{
ae-mail: mzstefaniak@gmail.com

${ }^{\mathrm{b}}$ Relativistic Heavy-Ion Collider

${ }^{\mathrm{c}}$ Large Hadron Collider
} 


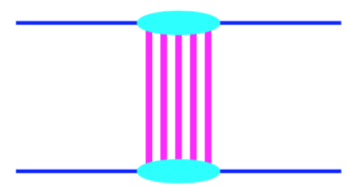

Figure 1: Hadron - hadron multiple scattering. Navy blue lines are hadrons and each of pink line illustrate Pomeron.[1]

EPOS is a generator based on Parton-Based Gribov-Regge Theory which is the mixture of two various theories. First one, Gribov-Regge theory uses effective field principies and it focuses on soft aspects of particle collision. The interactions are described with theoretical objects called Pomerons, which are crucial in calculation the cross-section in multiple scattering (Figure 1). The eikonalized parton model introduces quarks and gluons, what results with a more precise description of the interactions such as a gluon exchange. The $\mathrm{QCD}^{1}$ and $\mathrm{QED}^{2}$ are included in the description. [1] [2]

The combination of Gribov - Regge theory and parton model ensures that the energy is shared properly between both the particle production and the cross section. With the parton model there are hard processes added as well. The production of partons is represented by Lund model strings [3]. There are introduced objects called parton ladders. The open ones contribute to inelastic scattering which supports particles production. Meanwhile the closed ladders illustrates elastic scattering (Figure 2). In third version of EPOS there is applied the procedure consists hydrodynamic effects described in [4].

\section{Beam Energy Scan program}

Beam Energy Scan is a project run on the RHIC complex. The main goal, besides investigation of properties of QGP, is to understand the QCD phase diagram structure (Fig. [3]). Only a few pieces of information have been discovered yet. The QCD phase diagram is being mapped out in order to look for the evidences of:

- critical point fluctuations

- signals of $1^{\text {st }}$ order phase transition

- turn-off the signatures of QGP [5]

The gold ions are collided with such energies as: $\sqrt{s_{N N}}=7.7,11.5,19.6,27,39,62.4 G e V$.

One of the tools used with a view to studying occurring transitions of matter is femtoscopic correlations.

\section{Femtoscopy correlations}

\subsection{Two-particle correlations}

The method inspired by $\mathrm{HBT}^{3}$ interferometry [7] focuses on the smallest sizes in nature, about $10^{-15} \mathrm{~m}$ (femtometre), the sizes of nuclei. Studies of two-particle correlations allows one to examine space-time extension of the emitting source created during the collisions of nuclei. The size of area where the given kind of particles are produced is called homogeneity length [8].

\footnotetext{
${ }^{1}$ Quantum Chromodynamics

${ }^{2}$ Quantum Electrodynamic

${ }^{3}$ R. Hanbury-Brown and R. Q. Twiss
}

a)

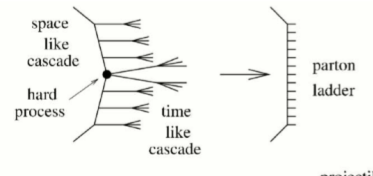

b)

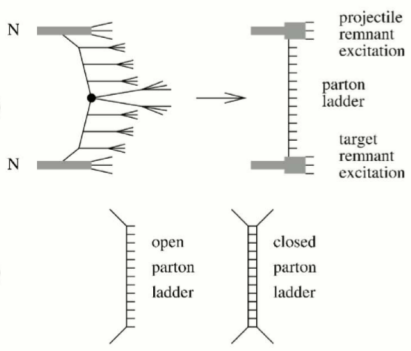

Figure 2: a) Elementary parton - parton scattering and representation as parton ladder. b) Full presentation of scattering, completed with remnants. c) Open and closed parton ladder [2] 


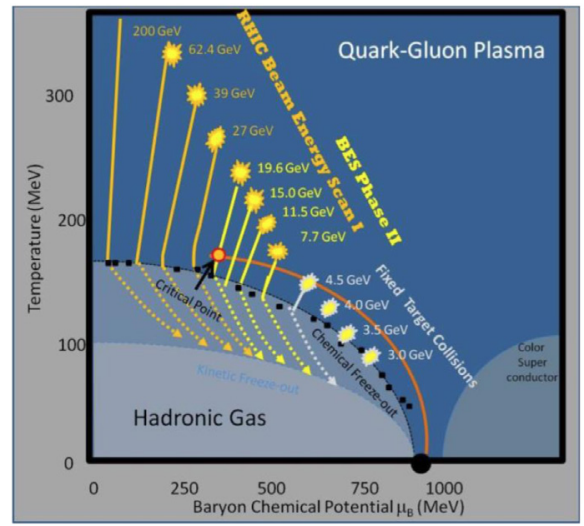

Figure 3: Studies of phase diagram peformed in a frame of BES program [6]

\subsubsection{Correlation function}

A two-particle correlation function defines ratio between probability of detecting two particles with given momenta and position simultaneously to product of detecting them separately [8].

$$
C\left(p_{1}, p 2\right)=\frac{P_{2}\left(p_{1}, p_{2}\right)}{P_{1}\left(p_{1}\right) P_{1}\left(p_{2}\right)}
$$

The source is often treated as a three-dimensional sphere and described with parametrization proposed by G. Bertsch and S. Pratt. The correlation function is represented in the -out -side -long (o-s-1) coordinate system [9]. Kopylov and Podgoretsky proposed the three-vector $\vec{q}=\overrightarrow{p_{1}}-\overrightarrow{p_{2}}$ so that the correlation function is represented in the following form:

$$
C\left(q_{\text {out }}, q_{\text {side }}, q_{\text {long }}, \lambda\right)=1+\lambda \exp \left(-q_{\text {out }}^{2} r_{\text {out }}^{2}-q_{\text {side }}^{2} r_{\text {side }}^{2}-q_{\text {long }}^{2} r_{\text {long }}^{2}\right)
$$

where $r_{\text {out,side, long }}$ are lengths of the source in out, side and long directions (three-dimensional femtoscopy radii) and $\lambda$ is a chaosity parameter [Fig. 4].

The out-direction width of the correlation function is inversely proportional to the lifetime of source (duration of particle emission). The side-direction measurements are inverse to the transverse size of source. The $R_{\text {side }}$ and $R_{\text {out }}$ are only proportional to the system average size or lifetime. The measurements of radii are affected by hydrodynamical flow or it depends on the chosen reference frame. In order to make such effects more irrelevant or even cancel them out, one can study the ratio $R_{\text {out }} / R_{\text {side }}$. The system size is approximately constant, while the lifetime of source varies depending on presense of a phase transition. Hence, the $R_{\text {out }} / R_{\text {side }}$ is considered to be optimal in studies of the

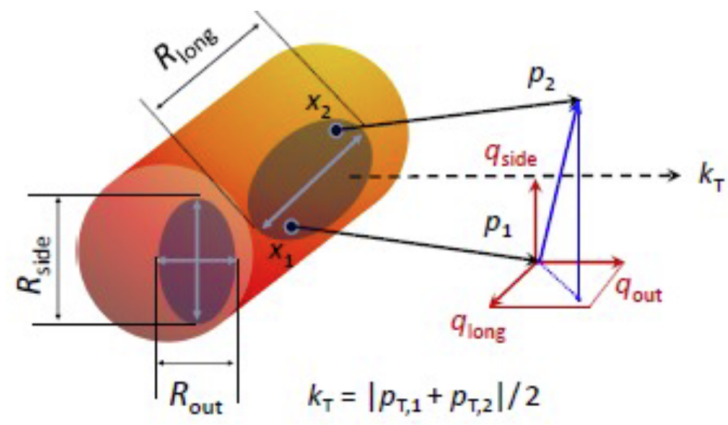

Dealing with experimental data one has to parametrize the two-particle correlation function with equation:

$$
C_{2}(\vec{q})=\frac{A(\vec{q})}{B(\vec{q})}
$$

where A (signal) is a momentum distribution of particles built in the same event, while B (background) is a reference momentum distribution obtained from different events.

Figure 4: Definition the three-dimensional femtoscopy radii. 


\section{Azimuthal anisotropy}

The azimuthal anisotropy characterizes expansion of highly-compressed matter created in heavy-ion collisions [Fig. 5]. It is one of the most significant tool used in studying hot and dense matter, described more detailed in [11].

One of the standard methods used in order to calculate flow is event plane method. The crucial part is to estimate the reaction plane from the measured particles. It is defined as a event plane in range $\Psi_{n}\left(-\pi / n \geq \Psi_{n}<\pi / n\right)$. For elliptic flow $n=2$ and in order to calculate event plane one can use

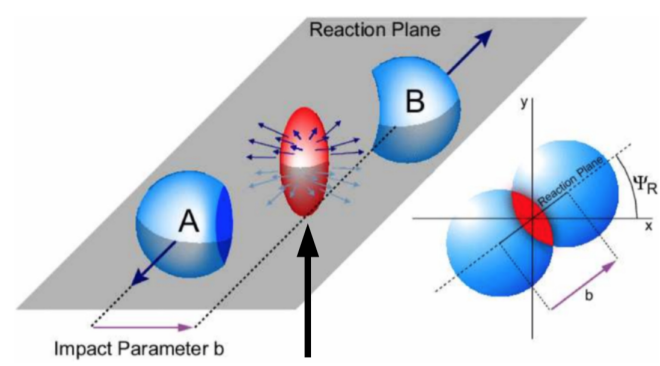

Figure 5: Nucleus after collision. equation:

$$
\Phi_{2}=\tan ^{-1}\left(\frac{\sum_{i} w_{i} \sin \left(2 \phi_{i}\right)}{\sum_{i} w_{i} \cos \left(2 \phi_{i}\right)}\right) / 2
$$

where: $i$ - selected particle, $\phi_{i}$ - azimuthal angle of particles, $w_{i}$-weight of $i$ particle [12]. In proposed $\eta-s u b$ method, from all measured particles there are selected two groups with "forward" and "backward" pseudorapidity with a gap between them. This method is introduced with a view to reducing the effect of "non flow" (e.g. decay of resonances to several charged daughter particles, jets or HBT correlations). [13]

To express the observed $v_{2}$ of particles with respect to already investigated event plane one uses:

$$
v_{2}^{o b s}\left(p_{T}, y\right)=\left\langle\cos \left[2\left(\phi_{i}-\Phi_{2}\right)\right]\right\rangle
$$

This is average over all particles in all events. As a consequence of final multiplicity limitation in the investigation of the angle of the reaction plane, the correction of $v_{2}$ with event plane resolution have to be done.

$$
R_{2}=\sqrt{\left\langle\cos \left[2\left(\Phi_{2}^{A}-\Phi_{2}^{B}\right)\right]\right\rangle}
$$

where: $\Phi_{n}^{A}$ - event plane calculated only using "forward-pseudorapidity" particles while $\Phi_{n}^{B}$ - with "backward-pseudorapidity" ones. The final result of $v_{2}$ calculation is given by:

$$
v_{2}=\frac{v_{2}^{o b s}}{R_{2}}
$$

\section{Results}

There were three different kinds of analyze performed: one-particle analyze - $p_{T}$ spectra, azimuthal anisotropy $-v_{2}$, two-particles analyze - femtoscopy correlations, in order to compare simulated and experimental data.

\subsection{Transverse momentum spectra}

Transverse momentum distribution is one of the elementary tools in High-Energy analyze. In this examination it was used to make basic comparison between simulated and experimental data published in [14]. Such measurements were done for $\mathrm{Au}+\mathrm{Au}$ collisions at two centrality ranges: $0-5 \%$ (central) and 60\% - 80\% (peripheral events). Particles with rapidity $|y|<0.5$ were taken into consideration. Below there are placed the obtained $p_{T}$ distributions for $\frac{h_{+}+h_{-}}{2}$, the sum of positive-charged hadrons $\left(p, \pi^{+}, K^{+}\right)$and negative once $\left(\bar{p}, \pi^{-}, K^{-}\right)$. 


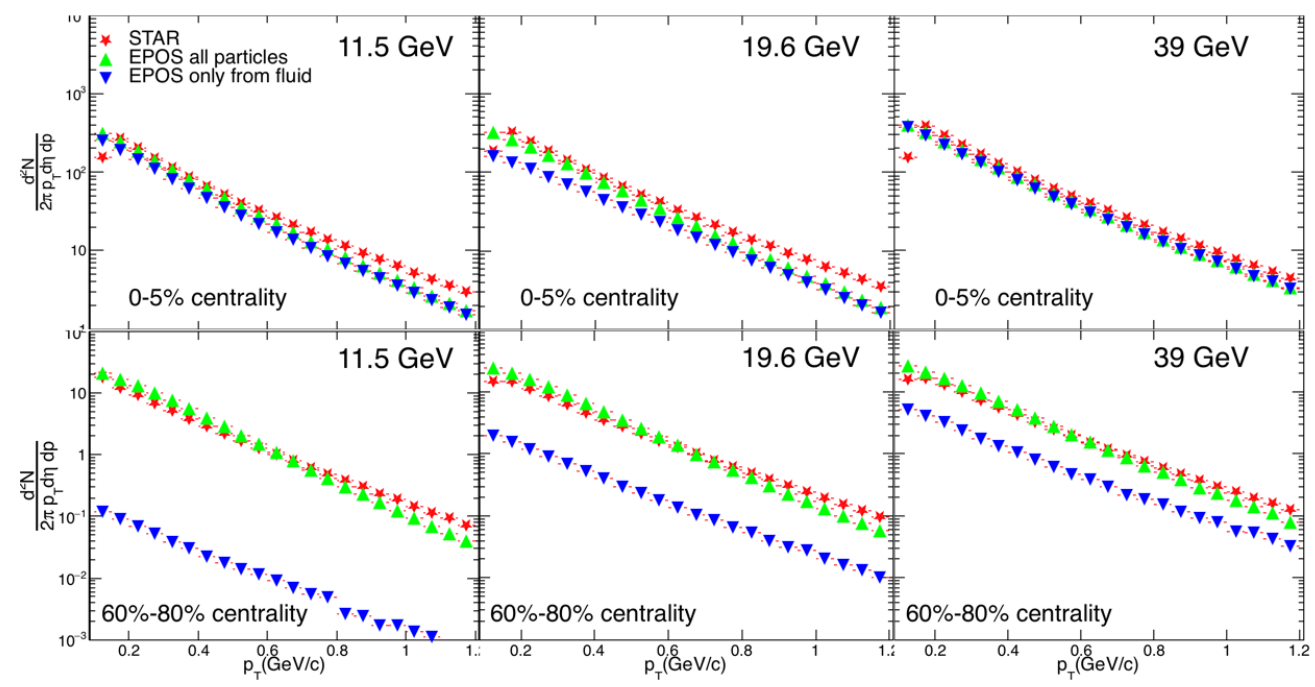

Figure 6: $p_{T}$ spectra for energies $\sqrt{s_{N N}}=11.5,19.6,39 \mathrm{GeV}$ at $0-5 \%$ and $60 \%-80 \%$ centrality ranges.

There is high agreement between experimental and theoretical data registered for the most central collisions. However, for lower energies for higher values of $p_{T}$ the slopes of plotted data are slightly different. In case of peripheral collisions EPOS data based on fluid equations values are considerably lower.

\subsection{Correlation function}

In this research there was performed positively charged pions femtoscopic analysis in $\mathrm{Au}+\mathrm{Au}$ collisions. In order to study beam energy in femtoscopy the were calculated and parametrized three-dimensional correlation functions. The $\pi^{+}$pairs were taken into consideration at $\sqrt{s_{N N}}=11.5 \mathrm{GeV} 19.6 \mathrm{GeV}$ at different centralities or $k_{T}$ ranges. With a view to inferring size of emitting source the fitting procedure was used (equation 2). Obtained radii and $\lambda$ parameter from calculation of simulated data were compared with published experimental results and presented in different combinations.

With the decrease of energy of collision $\sqrt{s_{N N}}$ the differences becomes more relevant. Calculated values of $R_{\text {out }}$ are in the range of expectations. The duration of particle emission is similar for compared systems. In case of measurements of $R_{\text {side }}$ the experimental results are higher then the simulated once, especially for lower $k_{T}$ bins. The sizes of sources created in STAR are bigger

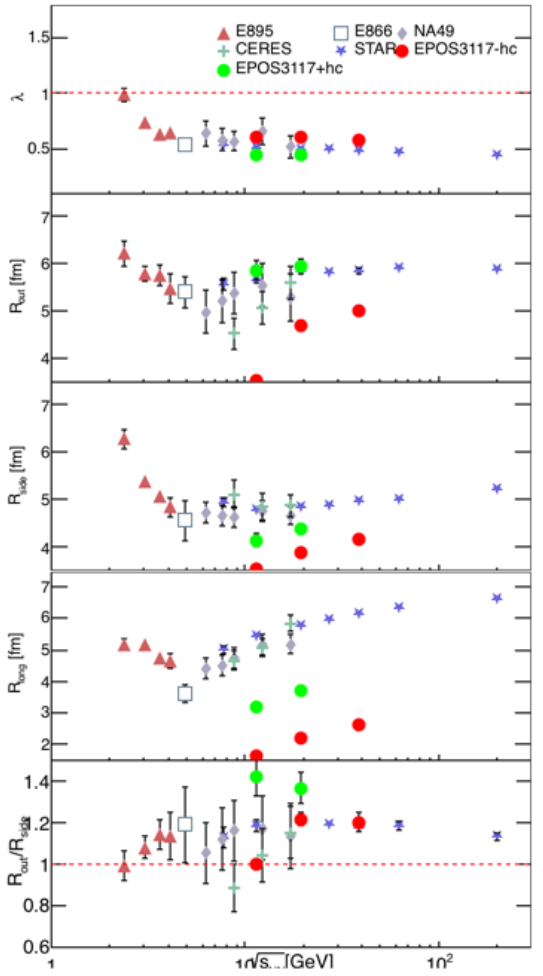

Figure 7: $\lambda$ and radii dependence on energy $\sqrt{s_{N N}}$ for different experiments and EPOS data $\left(k_{T} \approx 0.225 \mathrm{GeV} / \mathrm{c}\right)$. [15] 


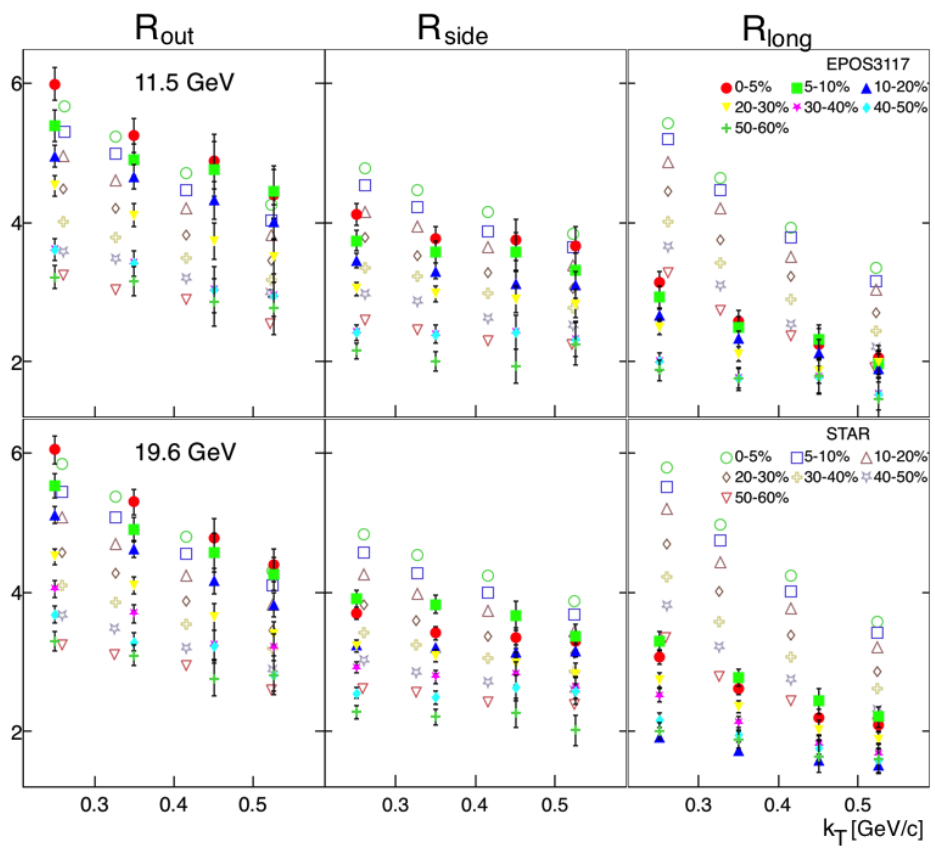

Figure 8: Radii calculated for various centralities and $k_{T}$ bins[15]

then simulated in EPOS. However, the most visible discrepancy is in studies of $R_{\text {long. }}$. In order to explain these differences the elliptic flow analyze was performed.

It is possible in EPOS generator to turn off some physics processes occur in heavy-ion collisions. In order to study the impact of hadron cascades on size of emitting source, they were turn off and turn on in simulation. The results are presented in Figure 7. Hadron cascades have relevant influence on values of calculated radii and $\lambda$ parameter. When they are present the size of the source is bigger and more similar to experimental studies. The $\lambda$ parameter and $R_{\text {out }}$ are in the range of expectations, while rest of parameters are considerably lower then in experimental results. Too high value of ratio $R_{\text {out }} / R_{\text {side }}$ means that the liftime of the source is shorter for simulated collisions than those obtained in experiments.

\subsection{Elliptic flow}

The studies of the elliptic flow were preformed in order to examine the description of matter expansion included in EPOS model. The data simulated with the EPOS were compared with STAR results published in [13]. The studies of beam energy were performed at mid-rapidity region for $p, \bar{p}, \pi^{+}$, $\pi^{-}, K^{+}, K^{-}$at $\sqrt{s_{N N}}=11.5,19.6,39 \mathrm{GeV}$ in minimum bias $(0-80 \%)$ using $\eta$-method . Particles with $|\eta|<1$, momentum between 0.15 and $5 \mathrm{GeV} / \mathrm{c}$ were taken into consideration. The calculated values for pions and kaons coming from simulation are slightly higher than experimental data. The same tendency is visible for antiprotons. The elliptic flow of protons in all $p_{T}$ bins are comparable for both data sets. 


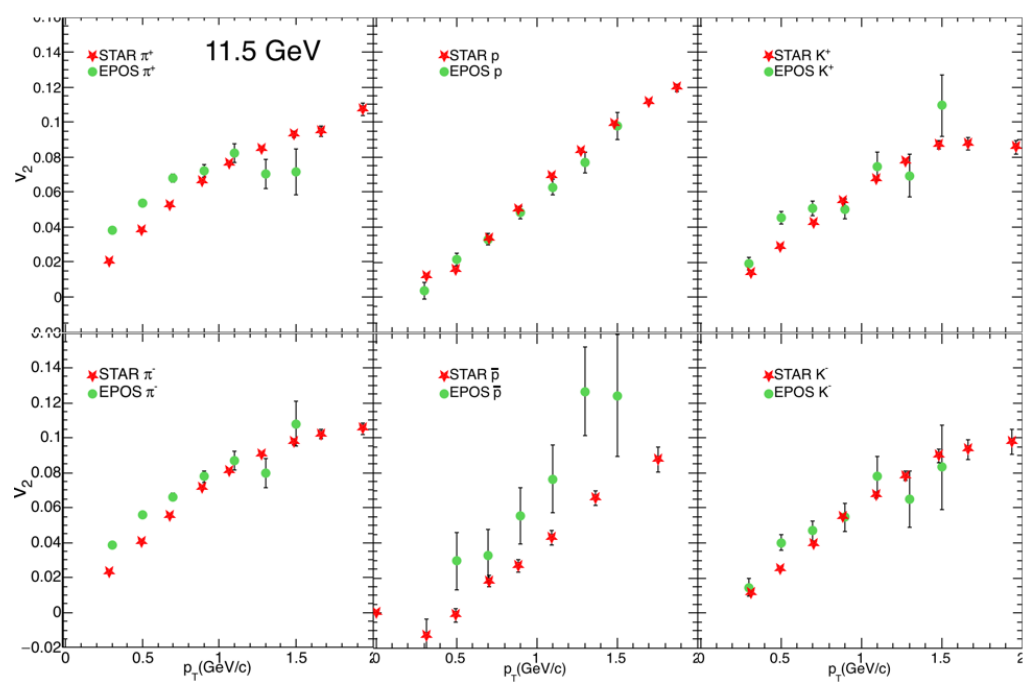

Figure 9: Elliptic flow dependence on $p_{T}$ [13].

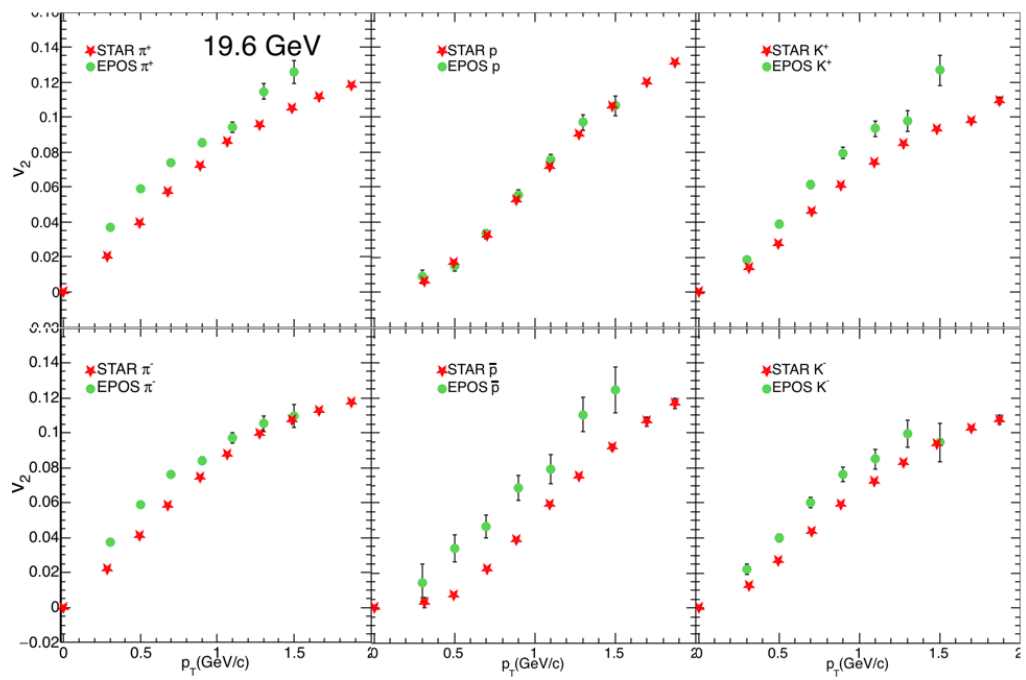

Figure 10: Elliptic flow dependence on $p_{T}[13]$.

\section{Summary}

The EPOS model predictions for Au+Au collisions at BES energy spectrum are verified with results obtained with experimental data. The $p_{T}$ spectra, correlation functions for identical pions and elliptic flow at 11.5 and $19.6 \mathrm{GeV}$ for both simulated and STAR data were compared and presented. Observed discrepancies can be caused by too small impact of fluid created in the collision. The transparency of the collision should be investigated as well. The work is still in progress. 


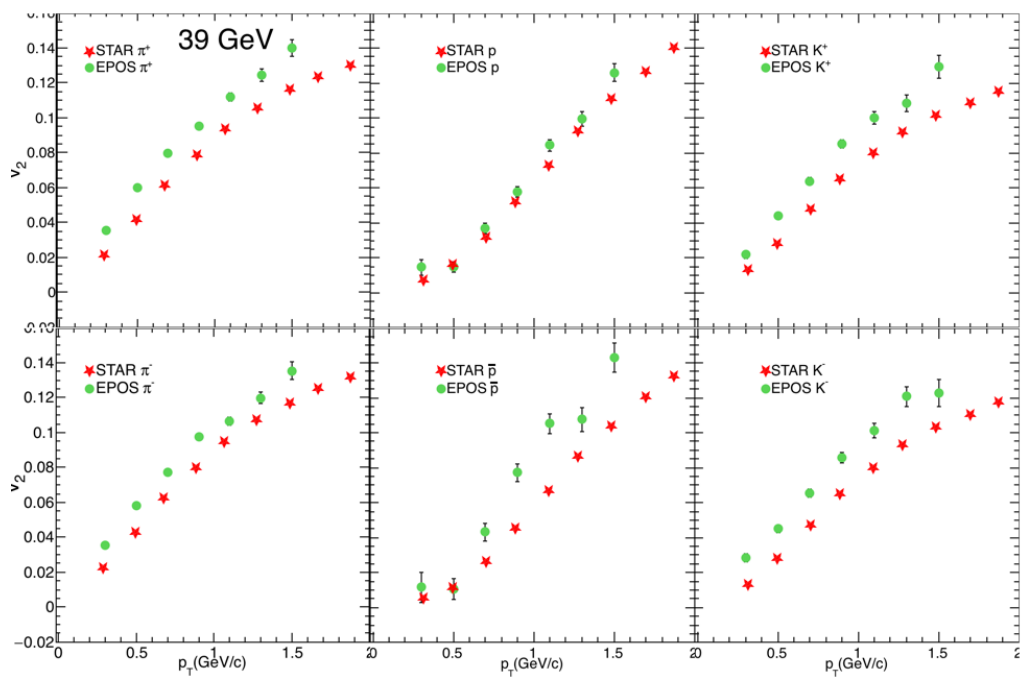

Figure 11: Elliptic flow dependence on $p_{T}[13]$.

\section{References}

[1] H.J. Drescher, M. Hladik, S. Ostapchenko, T. Pierog, K. Werner, Phys. Rept. 350, 93 (2001), hep-ph/0007198

[2] K. Werner, F.M. Liu, T. Pierog, Phys. Rev. C74, 044902 (2006), hep-ph/0506232

[3] M. Anderson, B. Pennington (1985)

[4] K. Werner, I. Karpenko, M. Bleicher, T. Pierog, S. Porteboeuf-Houssais, Phys. Rev. C85, 064907 (2012), 1203.5704

[5] G. Odyniec, "results and future of beam energy scan program at rhic"

[6] D. Cebra, "exploring the qcd phase diagram: Rhic beam energy scan ii", http://www.c-ad.bnl. gov/ardd/LEeC/Presentations/Cebra_LEReC_BESII_ver2.pdf, accessed: 2016-08-16

[7] U. Gordon Baym, University of Illinois, Hanbury brown - twiss intensity interferometry: from stars to nuclei to atoms and electrons, http://ithes.science-server.com/content/images/ colloquium/pdf/Baym.pdf, accessed: 2016-10-18

[8] M.A. Lisa, S. Pratt, R. Soltz, U. Wiedemann, Ann. Rev. Nucl. Part. Sci. 55, 357 (2005), nucl-ex/0505014

[9] H. Tilsner, Ph.D. thesis, Heidelberg U. (2002), http://www . ub . uni-heidelberg . de/archiv/2474

[10] D.H. Rischke, M. Gyulassy (1996), 9606039v1

[11] H. Masui, A. Schmah, A.M. Poskanzer, Nucl. Instrum. Meth. A833, 181 (2016), 1212 . 3650

[12] S.A. Voloshin, A.M. Poskanzer, R. Snellings (2008), 0809. 2949

[13] L. Adamczyk et al. (STAR), Phys. Rev. C88, 014902 (2013), 1301. 2348

[14] S.P. Horvat (STAR), J. Phys. Conf. Ser. 446, 012017 (2013), 1303.7260

[15] L. Adamczyk et al. (STAR), Phys. Rev. C92, 014904 (2015), 1403.4972 